\title{
Safety of Maternal Immunization Against Pertussis: A Systematic Review
}

\author{
Caroline D'Heilly $\cdot$ Charlotte Switzer $\cdot$ Denis Macina
}

Received: May 8, 2019 / Published online: September 17, 2019

(C) The Author(s) 2019

\begin{abstract}
The WHO recommends vaccination of all children against pertussis. However, newborn infants remain vulnerable to infection. Pertussis vaccination during pregnancy has been introduced in several countries to protect newborns via transplacental transfer of maternal pertussis antibodies to the infant. We reviewed the impact of maternal pertussis vaccination on the health of pregnant women, the developing fetus, and health of the newborn. We searched PubMed/MEDLINE, EMBASE, Scopus (Elsevier), Cochrane Database of Systematic Reviews, ProQuest, and Science Direct to identify studies that assessed the safety of maternal pertussis vaccination. Twenty-seven English language publications published between January 1995 and December 2018 were included in this review. Pregnant women receiving pertussis vaccines did not have increased rates of systemic or local reactions. There were no safety concerns with repeat vaccination with other tetanus-containing vaccines or their
\end{abstract}

Enhanced Digital Features To view enhanced digital features for this article go to https://doi.org/10.6084/ m9.figshare.9742721.

C. D'Heilly · D. Macina ( $\square)$

Sanofi Pasteur, Vaccines Epidemiology and

Modeling, Lyon, France

e-mail: denis.macina@sanofi.com

C. Switzer

Sanofi Pasteur, Pharmacoepidemiology, Toronto, Canada concomitant administration with influenza vaccines. Maternal pertussis vaccination did not adversely affect pregnancy, birth or neonatal outcomes. This review confirms the safety of maternal pertussis vaccination during pregnancy.

Funding: Sanofi Pasteur.

Plain Language Summary: Plain language summary available for this article.

Keywords: Maternal immunization; Pertussis; Pregnancy; Vaccine; Whooping cough

\section{PLAIN LANGUAGE SUMMARY}

Newborn infants are particularly vulnerable to whooping cough in the first few months after birth. When women are vaccinated against whooping cough during pregnancy, their antibodies are transferred to the fetus, which protects the newborn infant from the disease. We summarized the published evidence of the safety of maternal immunization against whooping cough, both for the mother and infant. We found that vaccination against whooping cough was safe for both mothers and their newborn infants. Pregnant women were no more likely than non-pregnant women to experience injection site reactions or wholebody effects. There were no negative effects on the pregnancy, birth or the infant. Vaccination of pregnant women can be recommended for 
protection of vulnerable infants during their first weeks of life.

\section{INTRODUCTION}

Pertussis affects individuals of all ages but is especially concerning in infants, who account for most of the hospitalizations and deaths associated with the disease [1]. A modeling study based on World Health Organization (WHO) data from 2014 estimated that 24.1 million cases and 160,700 deaths could be attributed to pertussis among children aged $<5$ years. Of these, 5.1 million $(21 \%)$ cases and 85,900 (53\%) deaths occurred in infants aged $<1$ year [2].

To reduce pertussis-related morbidity and mortality in infants and young children, the WHO recommends acellular pertussis $(\mathrm{aP})$ or whole-cell pertussis (wP) vaccination for all infants, with the primary immunization series initiated at 6 weeks, and no later than 8 weeks of age [3]. However, infants are particularly susceptible to pertussis infection from multiple sources during the period between birth and start of their primary immunization series [4-7]. Studies have consistently documented high incidence rates and significant mortality in young infants [8-11]. For example, in the USA, the incidence of pertussis was $117.7 / 100,000$ person-years in infants aged $<1$ year between 2005 and 2010, with the highest rate in those aged 2 or 3 months (235.3/100,000 person-years or $247.7 / 100,000$ person-years, respectively) [8]. Elsewhere, pertussis-related deaths were estimated to be 0.6 per 100,000 maternities for infants aged $<66$ days in England during 2001 and 2011 [11]. The WHO does not currently recommend vaccination against pertussis before 6 weeks of age due to paucity of data on efficacy and safety, and lack of stand-alone aP vaccine $[3,12]$. Risk-management through cocooning, where close contacts of the infants are vaccinated, has had limited success [3].

Maternal immunization during pregnancy, taking advantage of transplacental transfer of pertussis antibodies $[1,3,12]$, was proposed to indirectly protect neonates against pertussis. This strategy achieves high pertussis antibody concentrations in infants [13], and is $64-93 \%$ effective in preventing disease among infants aged $<2$ months [14-21]. Moreover, breakthrough disease among infants whose mothers were vaccinated during pregnancy was less severe than those born to unvaccinated mothers [22]. The WHO also suggests that vaccination of pregnant women is likely to be the most effective and cost-effective strategy for disease prevention in infants too young to be vaccinated [3].

Some patients and healthcare providers are hesitant to or do not vaccinate during pregnancy because of perceived (vaccine) safety [23-25]. Other barriers to vaccination during pregnancy from the patient and healthcare perspective include negative media, missed vaccination opportunity (immunization not offered or requested), lack of vaccine stock, inadequate reimbursement, and limited patient interest [26-31]. Evidence-based educational programs that emphasize vaccine safety during pregnancy and protection against disease in infancy help support vaccine confidence and recommendations. To capture supporting evidence, we conducted a systematic review of the safety of maternal pertussis vaccination (Tdap and Tdap-IPV vaccines) during pregnancy.

\section{METHODS}

Studies assessing pertussis vaccination during pregnancy were identified from a systematic search of PubMed/MEDLINE, EMBASE, Scopus (Elsevier), Cochrane Database of Systematic Reviews, ProQuest, and Science Direct. We also scanned the reference lists of identified publications and searched ProQuest thesis, Clinicaltrials.gov, relevant conference and congress abstracts, and the Trial Trove database. The protocol for this systematic review was registered on PROSPERO (PROSPERO 2016:CRD42016038317) [32].

Our initial search was restricted to English language publications between January 1995 and June 2016. Further searches were performed to capture relevant studies published between July 2016 and October 2018. We used a combination of keywords that included the 
following: [1]: [(pertussis OR whooping cough) AND (vaccine OR Tdap OR immunization) AND (pregnancy OR pregnant OR pre-partum OR gestation OR maternal)], [2]: [1] AND (safety OR adverse event OR adverse reaction OR congenital abnormalities OR congenital disorder OR congenital malformation OR birth defects OR teratogens OR teratogenic agent OR teratogenicity OR pregnancy outcome OR pregnancy termination OR congenital anomaly OR developmental disorder OR adverse birth outcome).

PICOS (Patient Population or Problem, Intervention [treatment/test], Comparison [group or treatment], Outcomes, and Setting) criteria [33] were applied while considering interventional and observational studies in humans. Pregnant women and their offspring constituted the studied population. The intervention was pertussis immunization during pregnancy and the infant series of vaccination. Comparison groups were either no vaccination or standard of care vaccination, as well as pregnancy or no pregnancy. Outcomes reviewed were qualitative (nature, severity) and quantitative characterization of adverse events \{number, frequencies, and relative measures [e.g. odds ratios (OR) and relative risk (RR)]\} in pregnant women (including but not limited to local reactions and fever), in fetuses (including but not limited to spontaneous abortion, stillbirth, premature birth, birth weight, fetal growth, congenital malformation), and in infants prior to the first dose of primary immunization.

Reviews, meta-analyses, case reports, opinion pieces, letters to editors, and modeling studies were excluded. We also excluded studies on vaccination program improvement, vaccine uptake, vaccine acceptability and perception studies, and health economics studies.

A two-step process involving two independent reviewers at each step was employed to select studies for inclusion in the review. The first step included scanning titles and abstracts of retrieved references to select publications based on their relevance to our review. Publications of primary research studies reporting the safety of pre-partum pertussis vaccination were retained for full-text review. The second step consisted of a full-text review of the article to assess whether the report met the inclusion and exclusion criteria. Pre-structured MS Excel forms were used to extract relevant data from the included studies. Multiple factors including heterogeneity in study designs, vaccines used, outcomes measured, measurement/analysis methods, and the background pertussis incidence precluded a meta-analysis of the safety findings of the included studies.

This article is based on previously conducted studies and does not contain any studies with human participants or animals performed by any of the authors.

\section{RESULTS}

A total of 335 articles were identified from the comprehensive search criteria, of which 27 articles (summarized in Table 1) were included in this review (Fig. 1).

\section{Vaccine Reactogenicity in Pregnant Women}

Fifteen studies reported vaccine reactogenicity among pregnant women following immunization with Tdap or Tdap-IPV, among which nine were prospective clinical trials $[13,34-41]$ and six were database analyses [42-47] (Table 1). The most commonly reported reactions were injection site reactions, consistent with the Tdap label. While injection site reactions such as pain/tenderness, induration/swelling, itching, and erythema/redness were reported after Tdap/ Tdap-IPV administration in most clinical studies, pregnancy was not considered to have increased the rates of these events [13, 34, 36-38, 40]. However, moderate-to-severe injection site pain was more frequent in pregnant women than nonpregnant women in one study [34]. Injection site reactions assessed over 7 days were more common after Tdap than placebo in one small clinical study [13] but occurred at similar rates over $48 \mathrm{~h}$ in another slightly larger study [40]. Systemic and local reactions occurred at similar frequencies in pregnant women receiving Tdap and those receiving tetanus vaccines in two clinical studies $[35,36]$. 


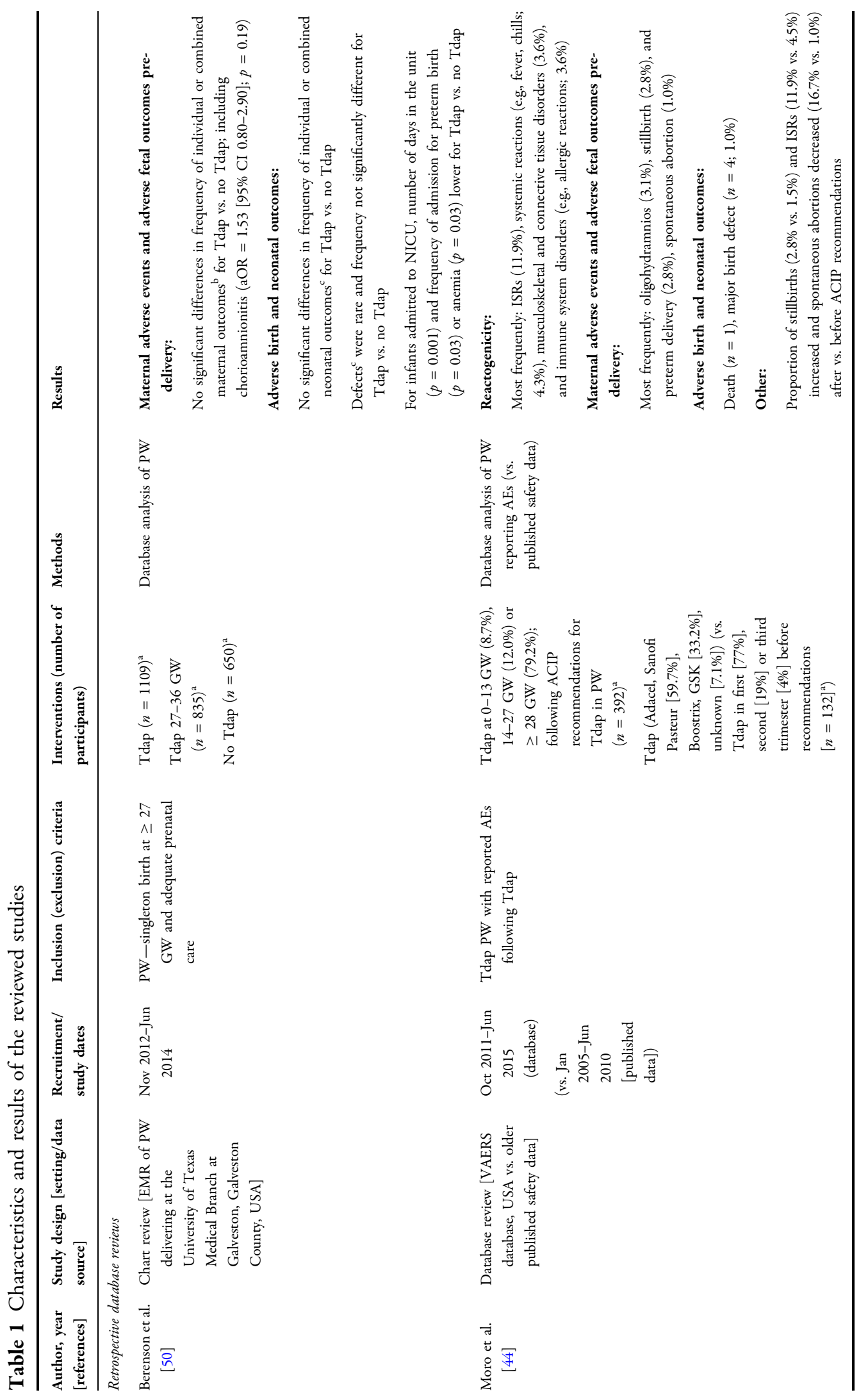




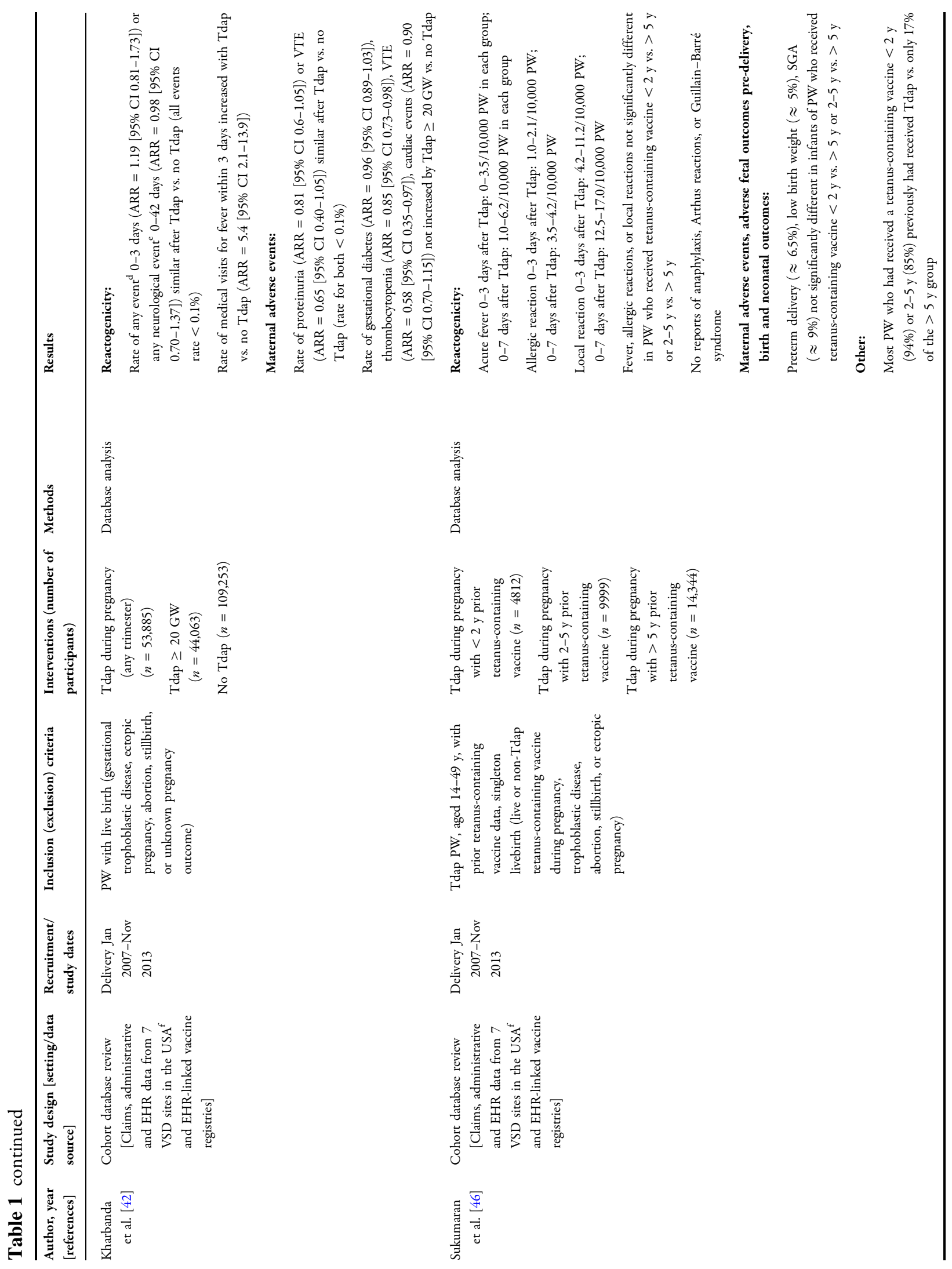




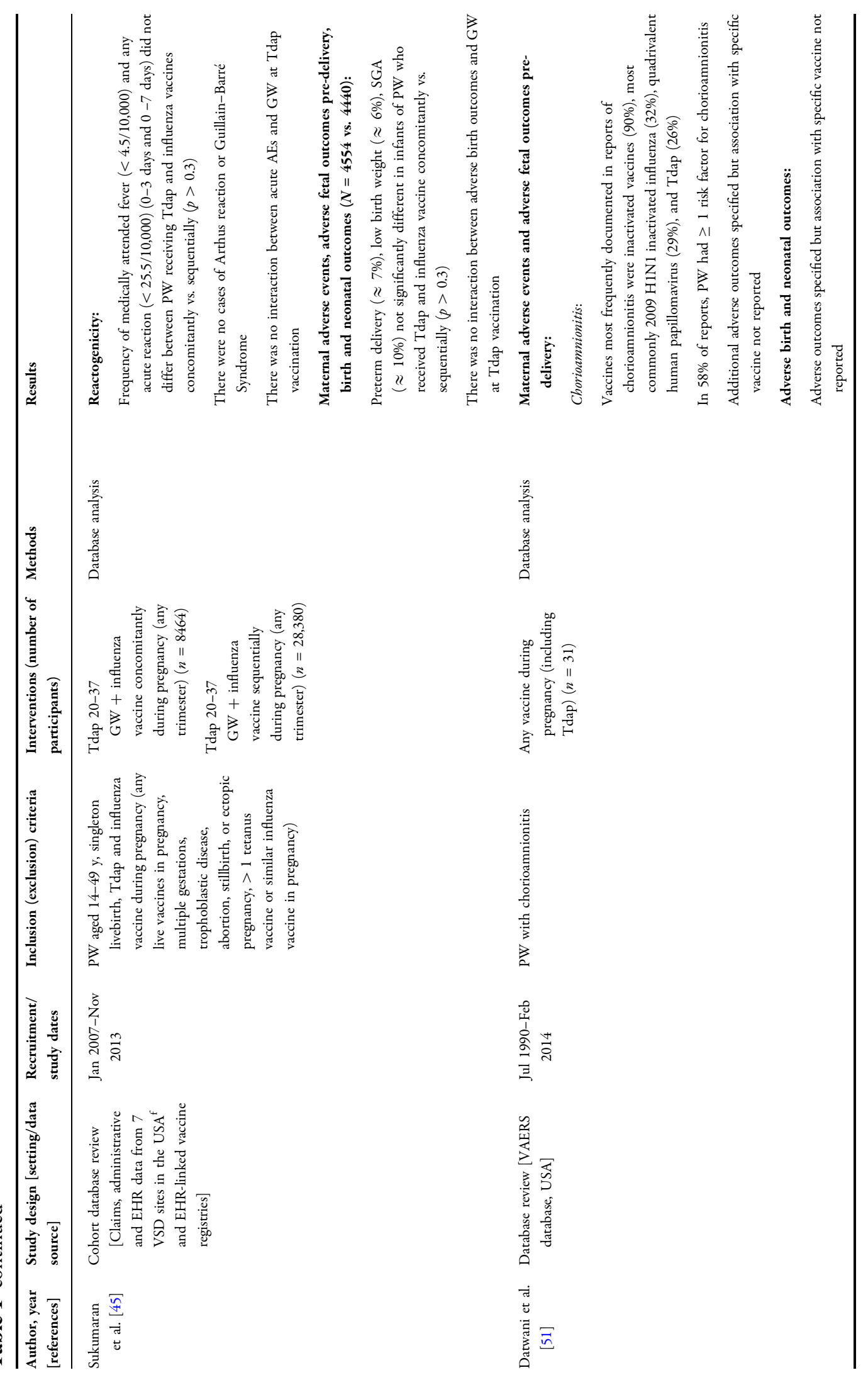




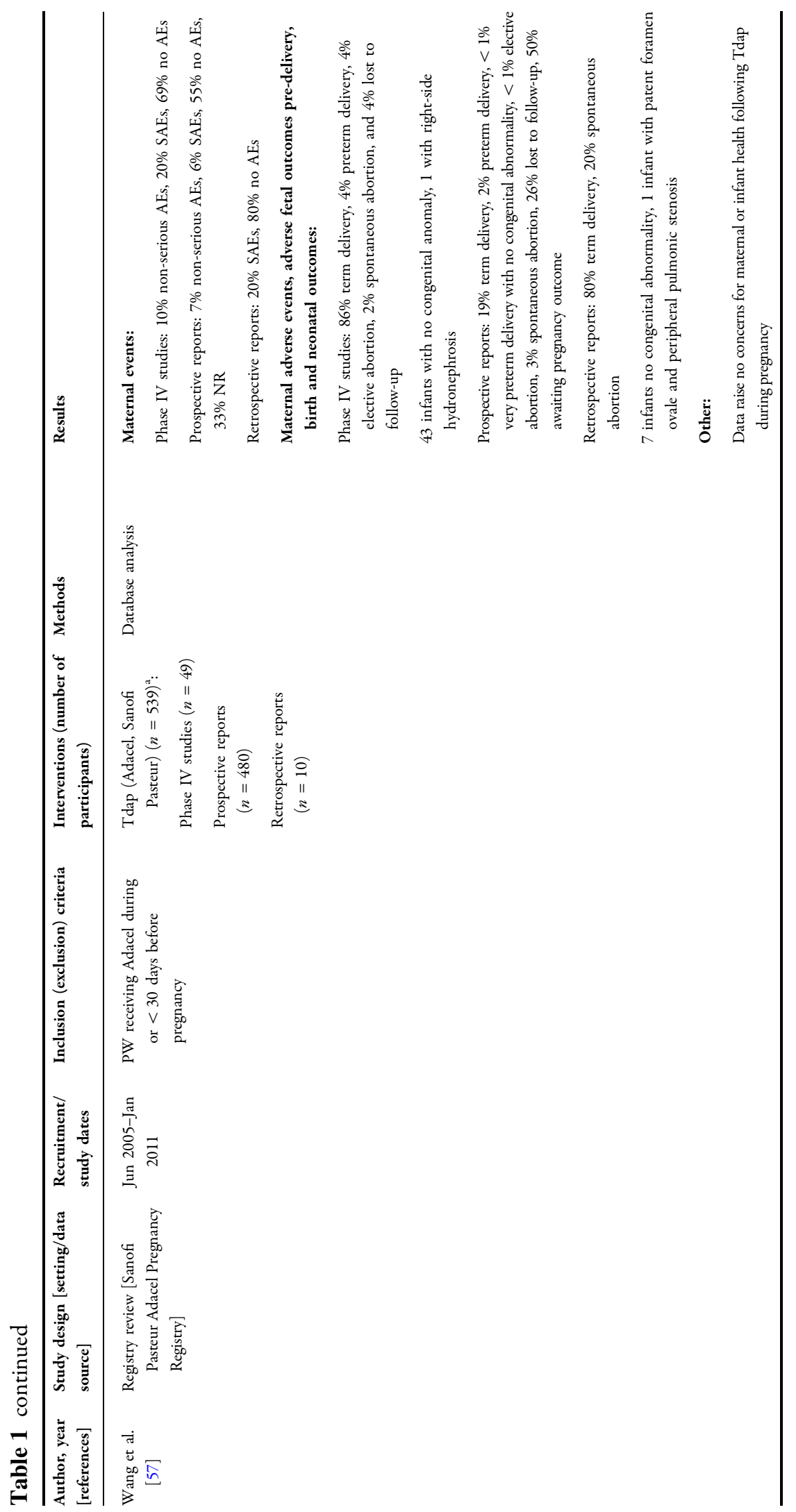




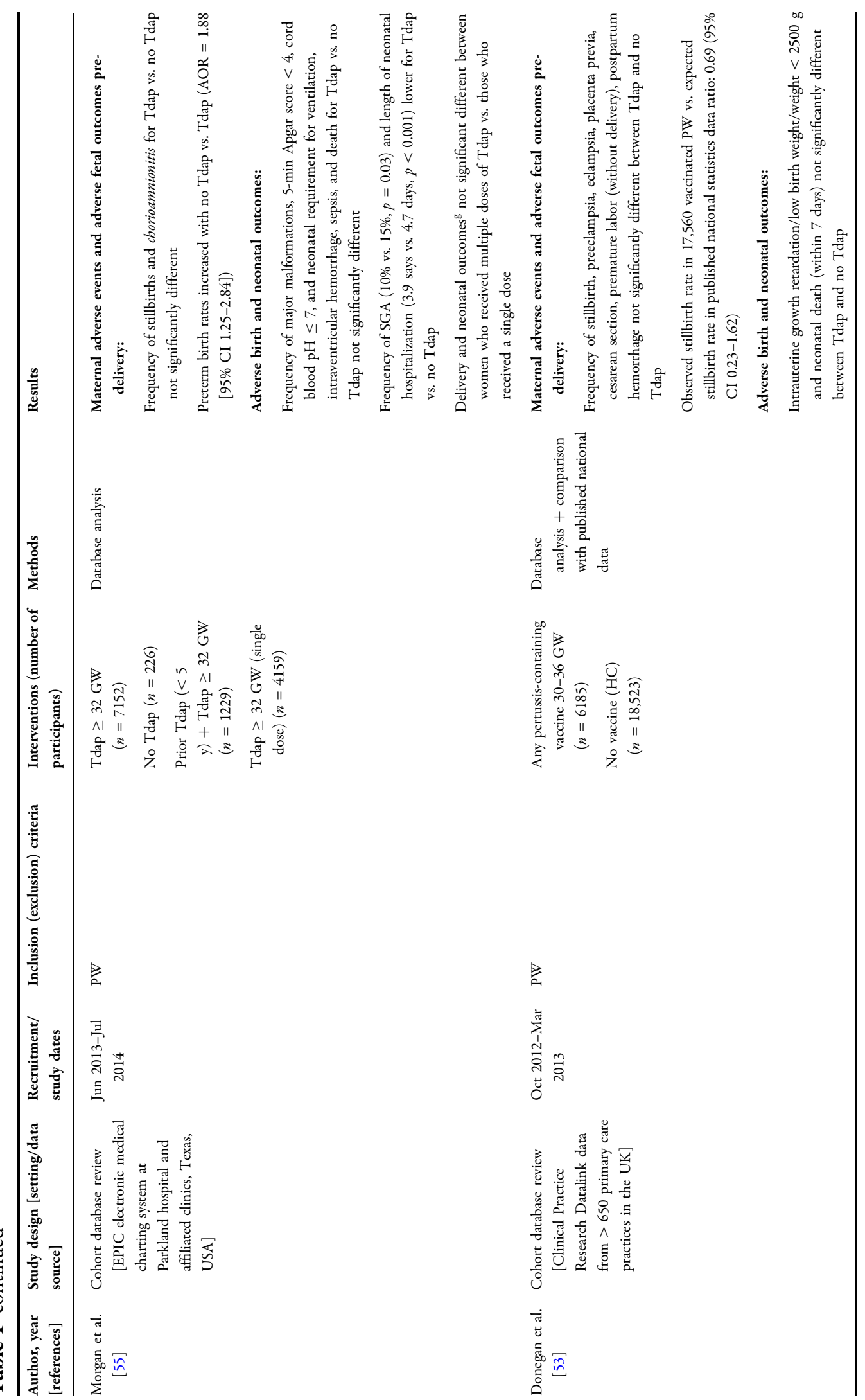




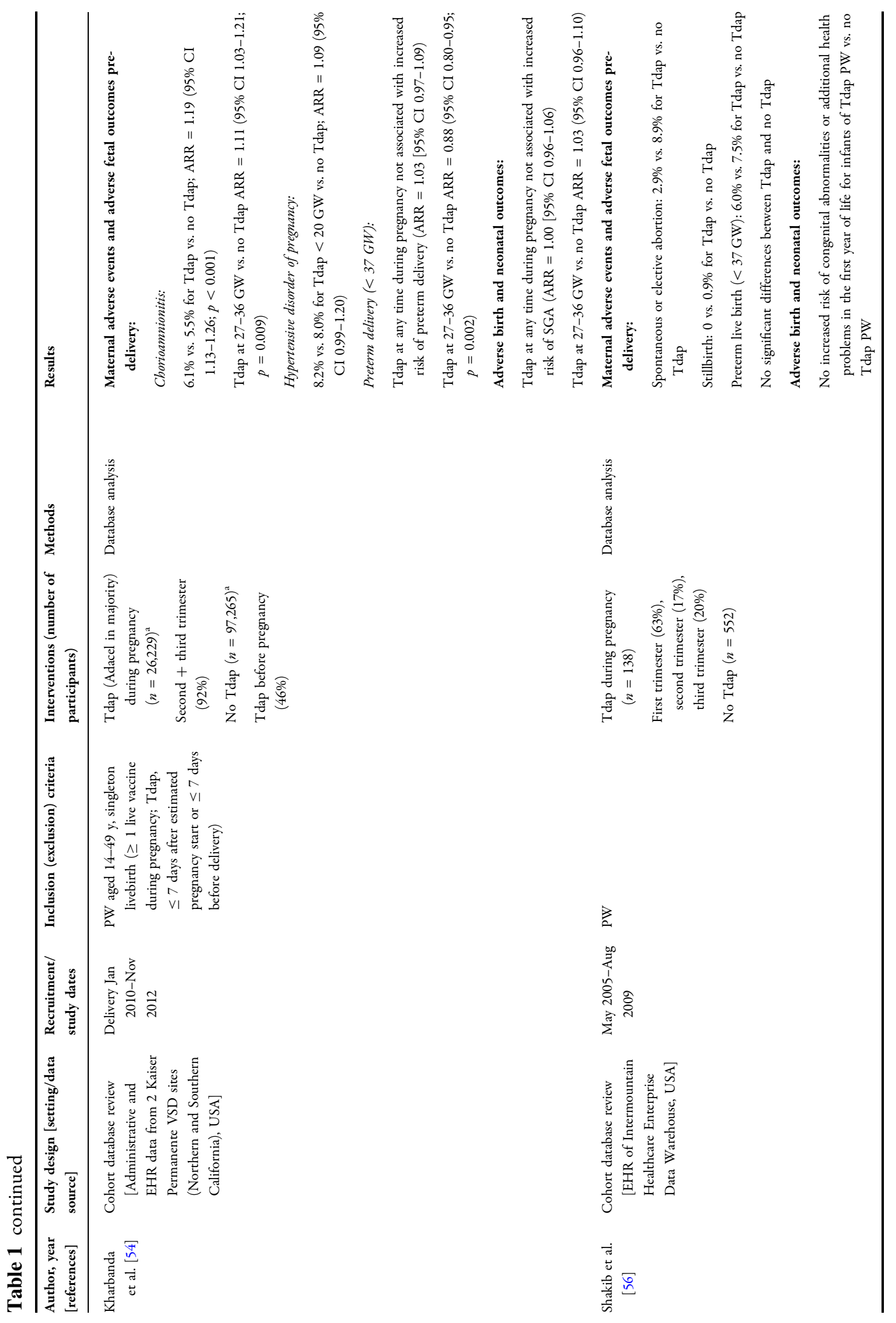




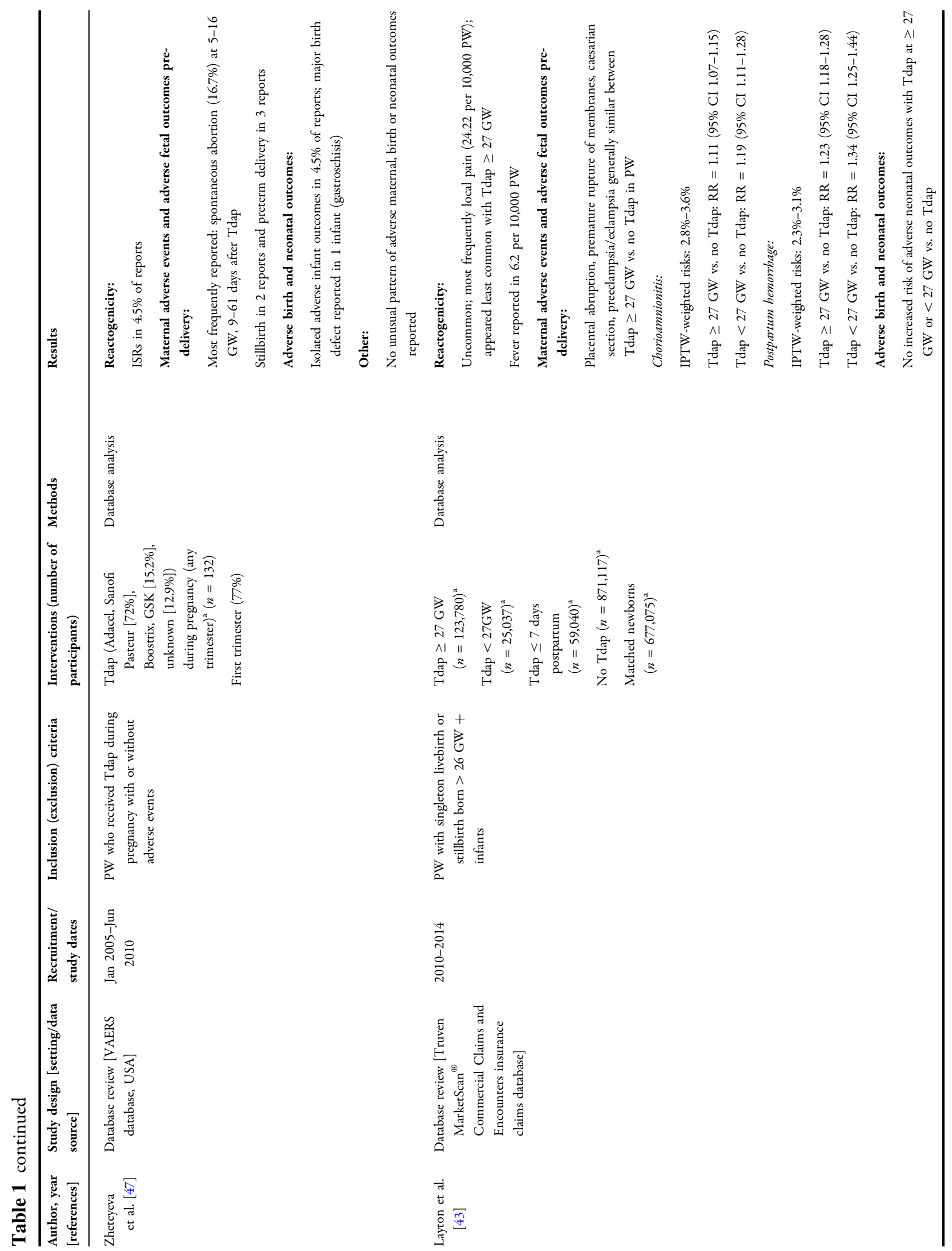




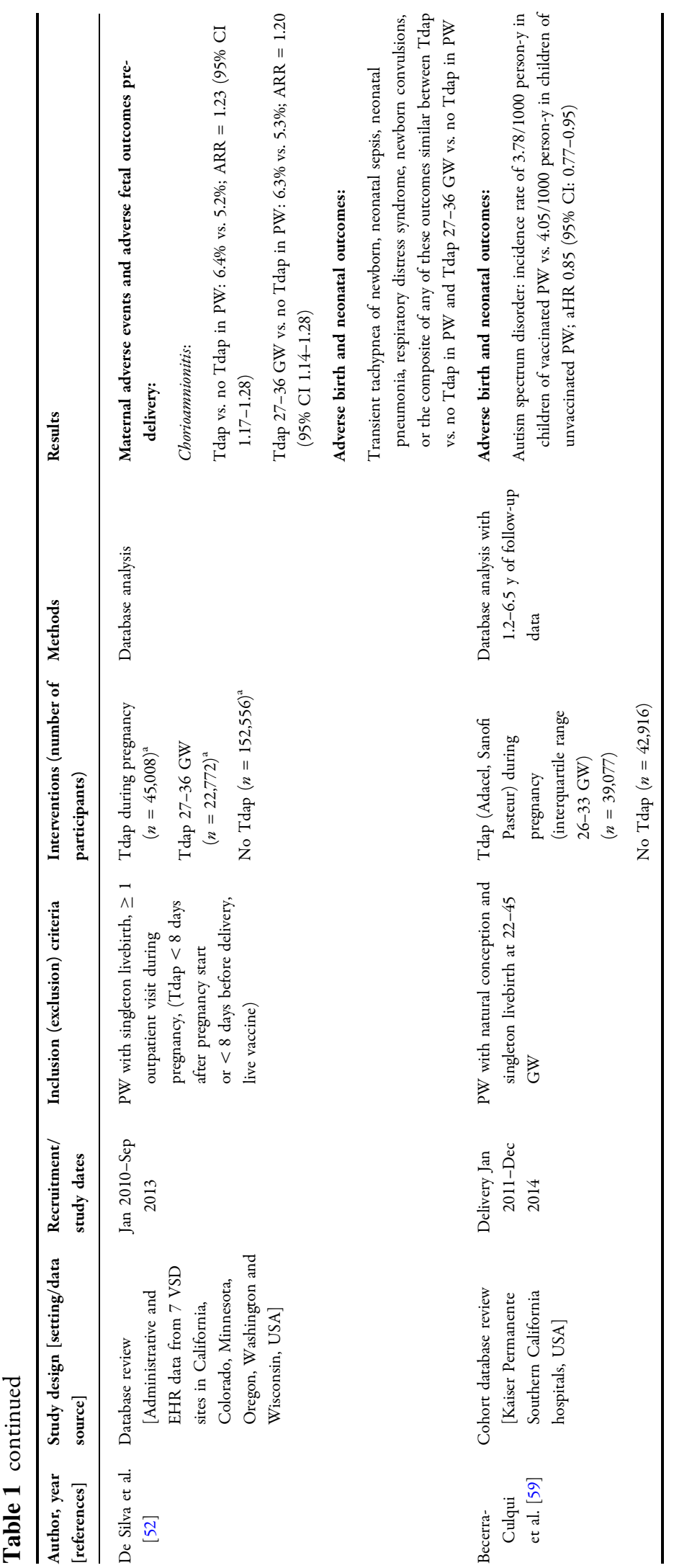




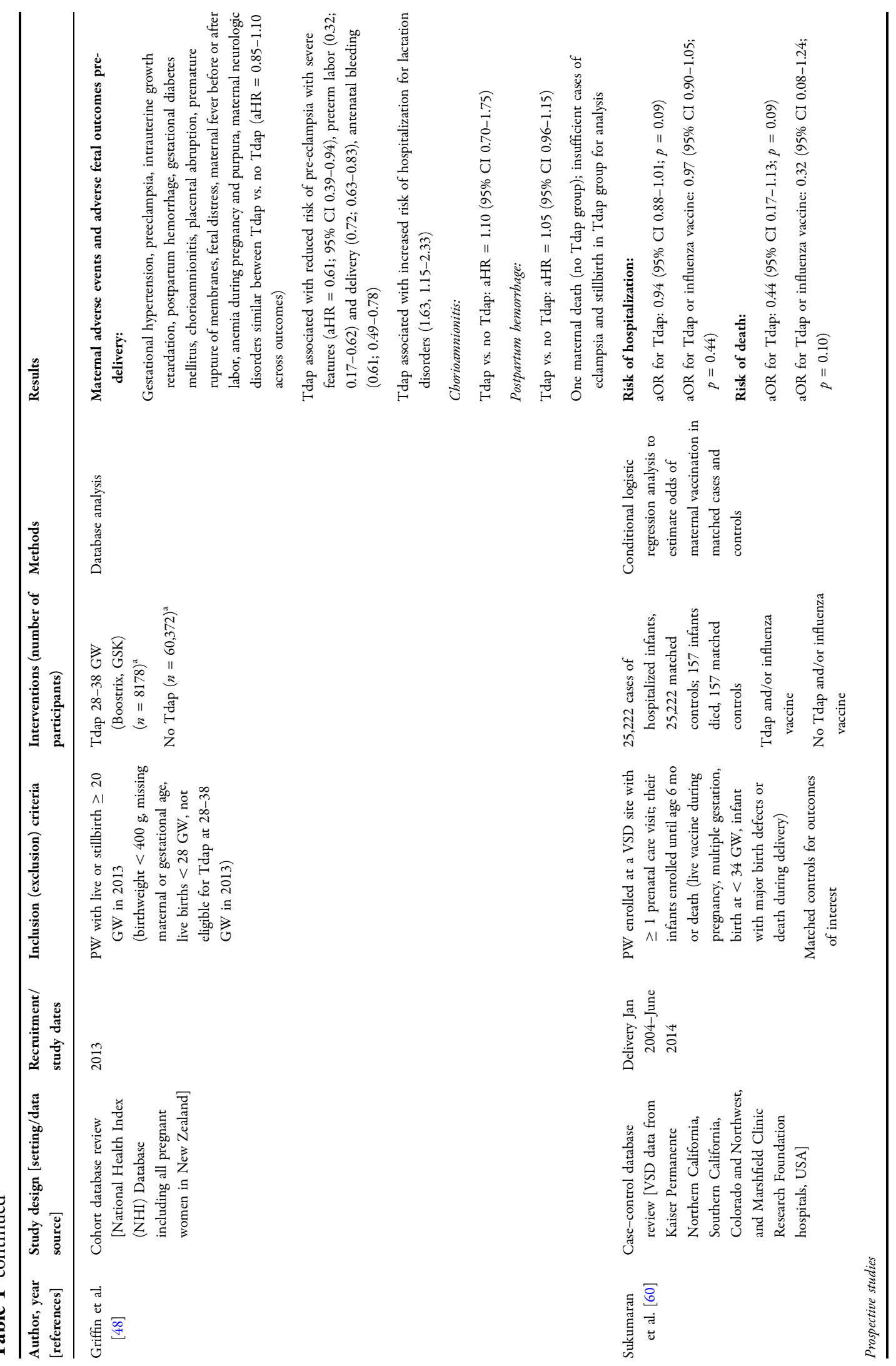




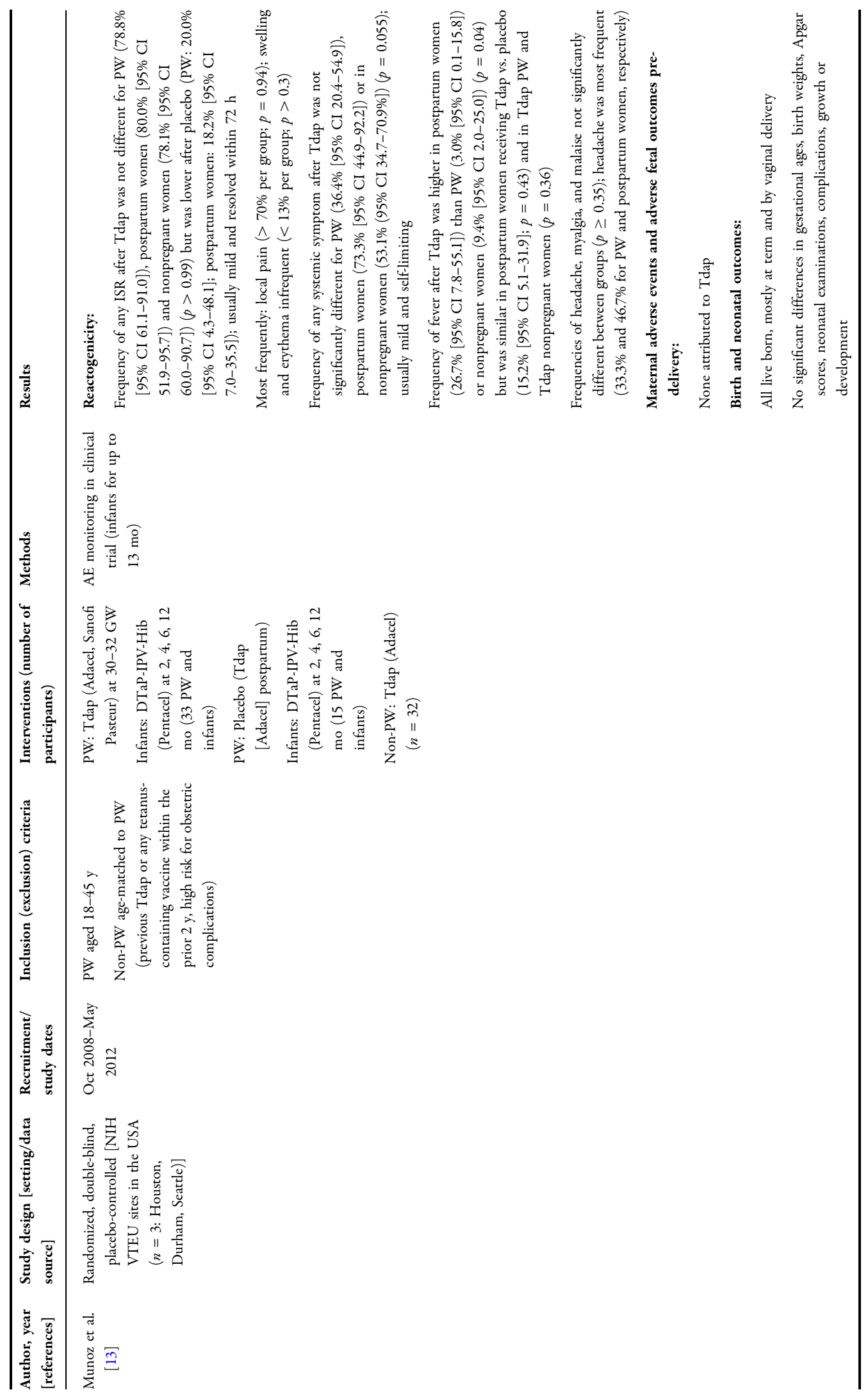




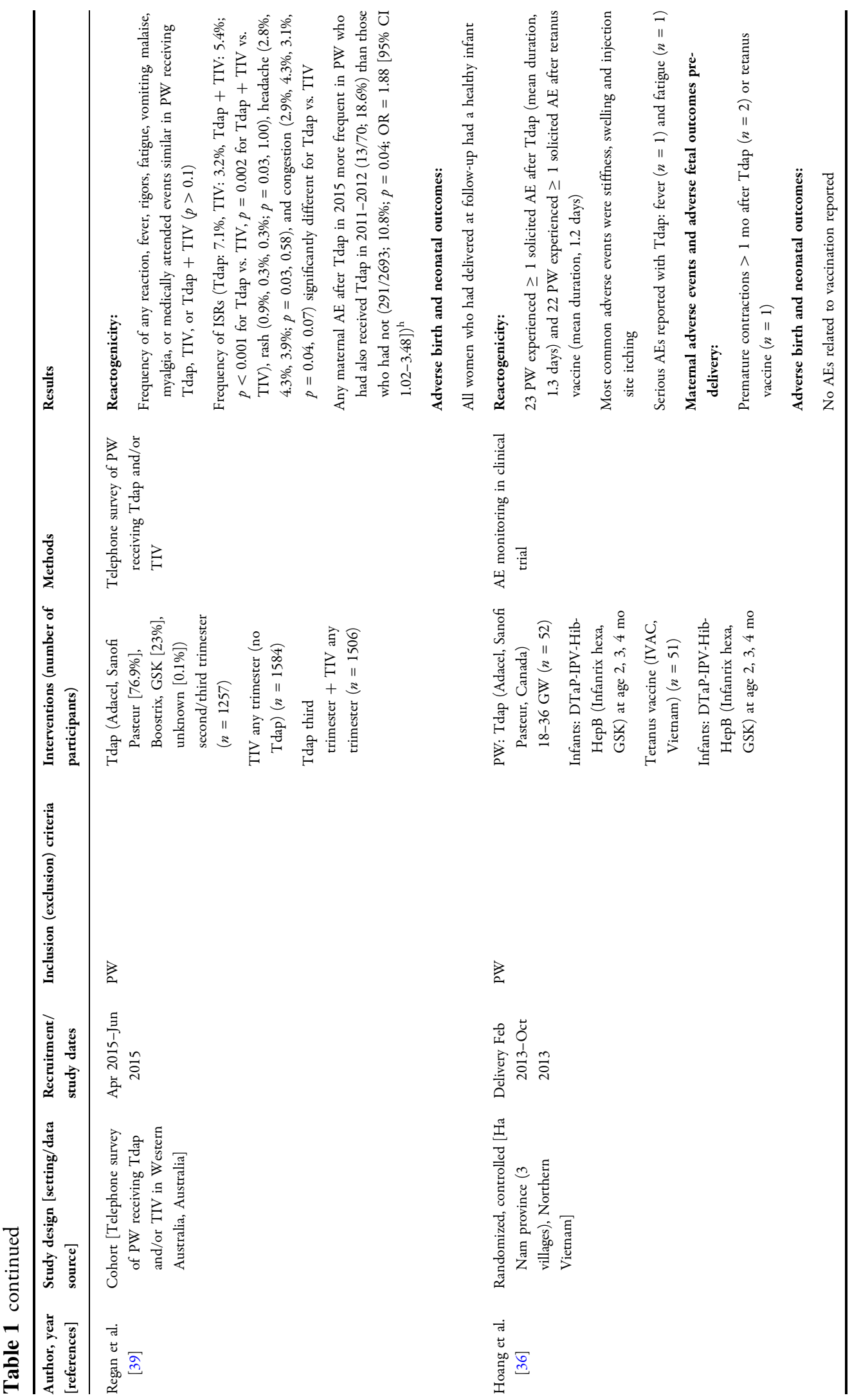




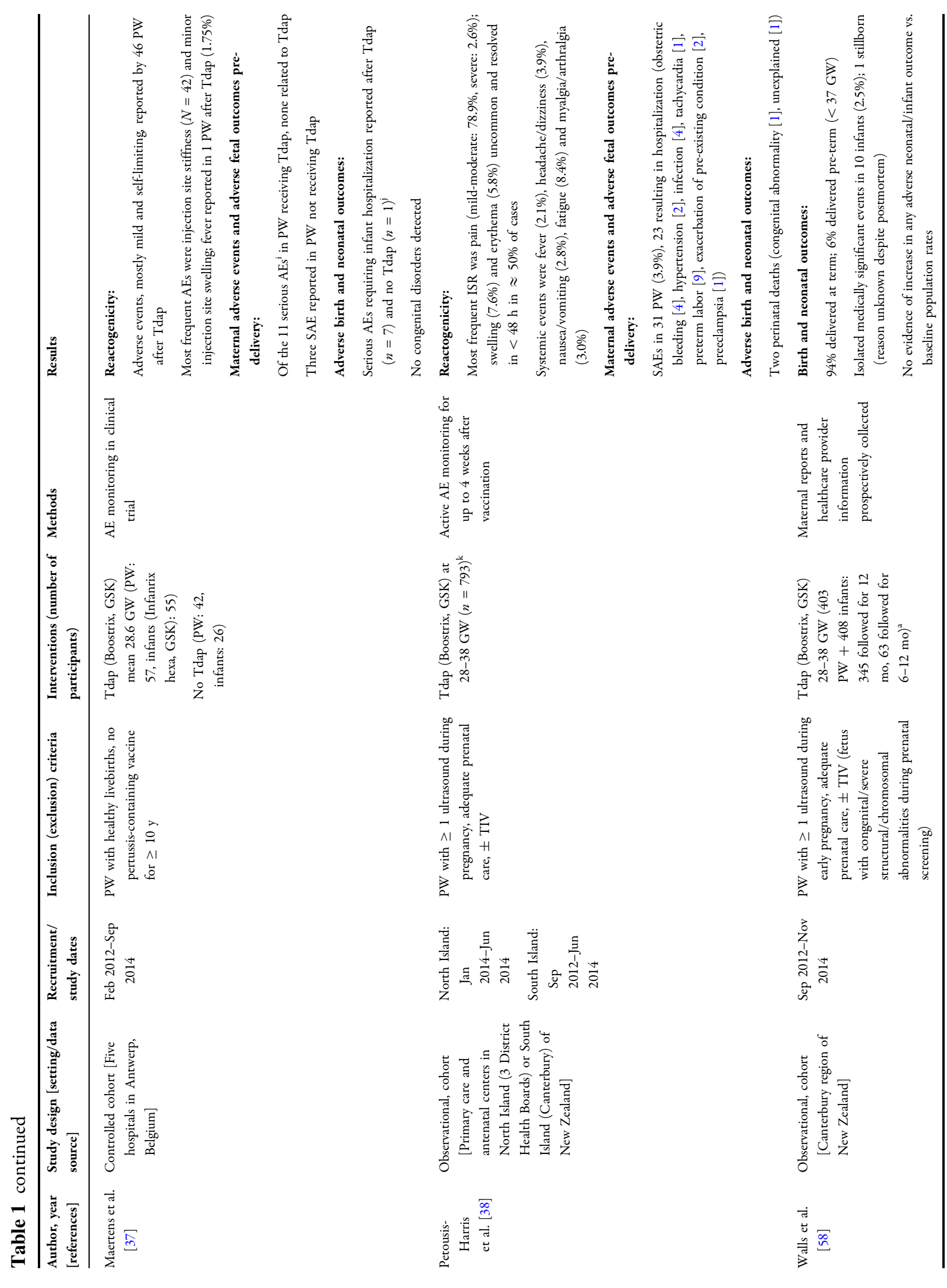




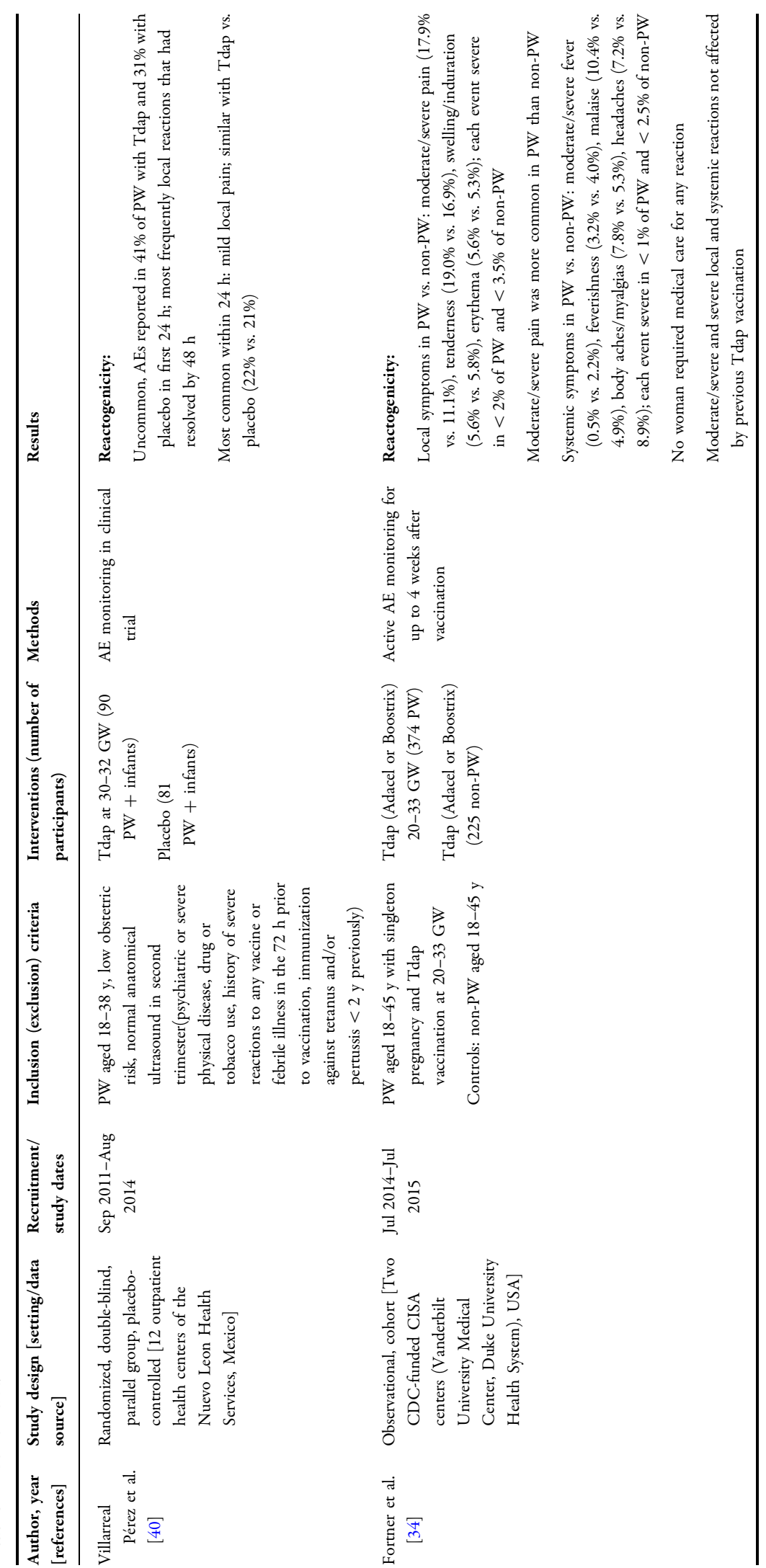




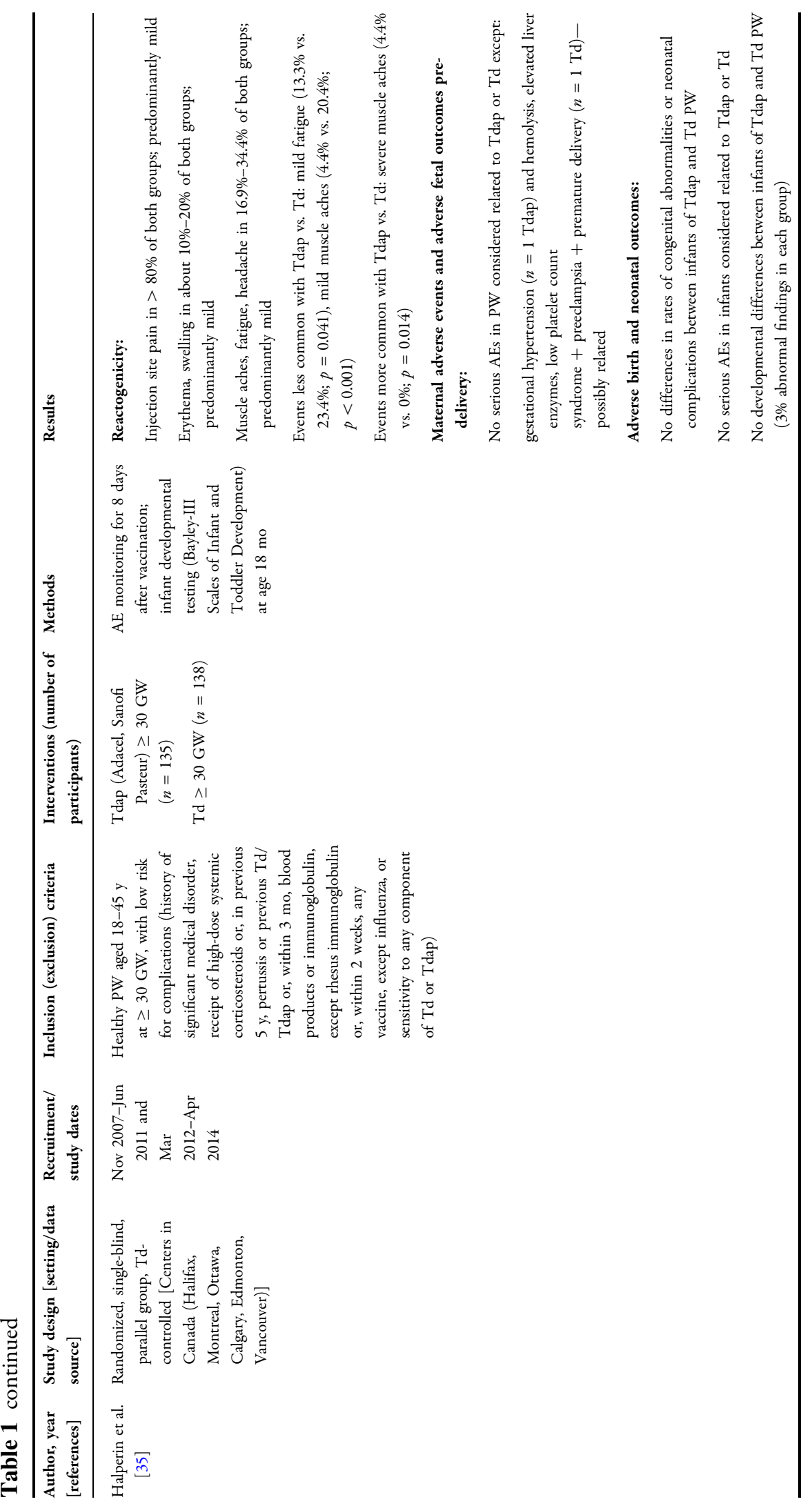




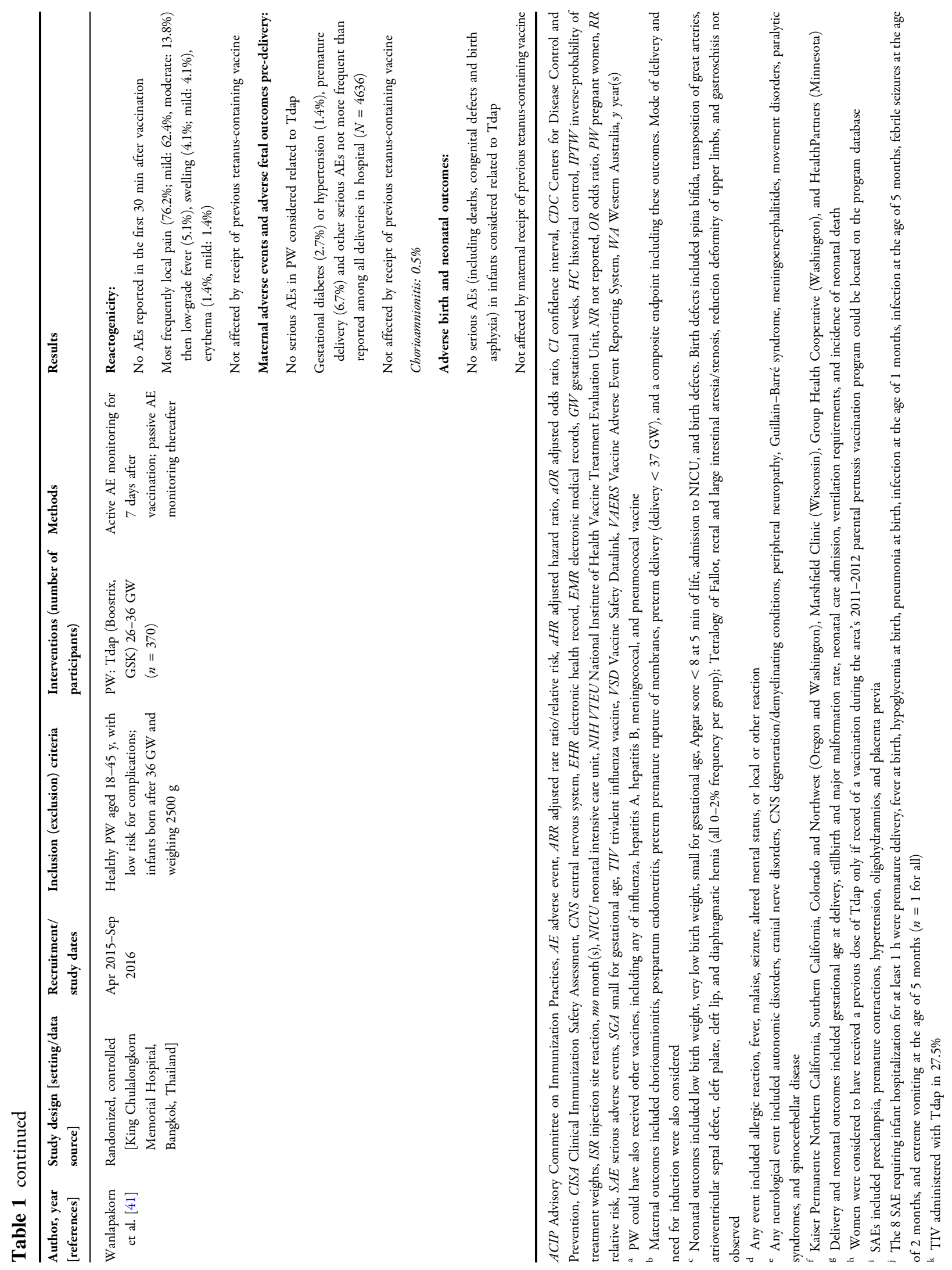




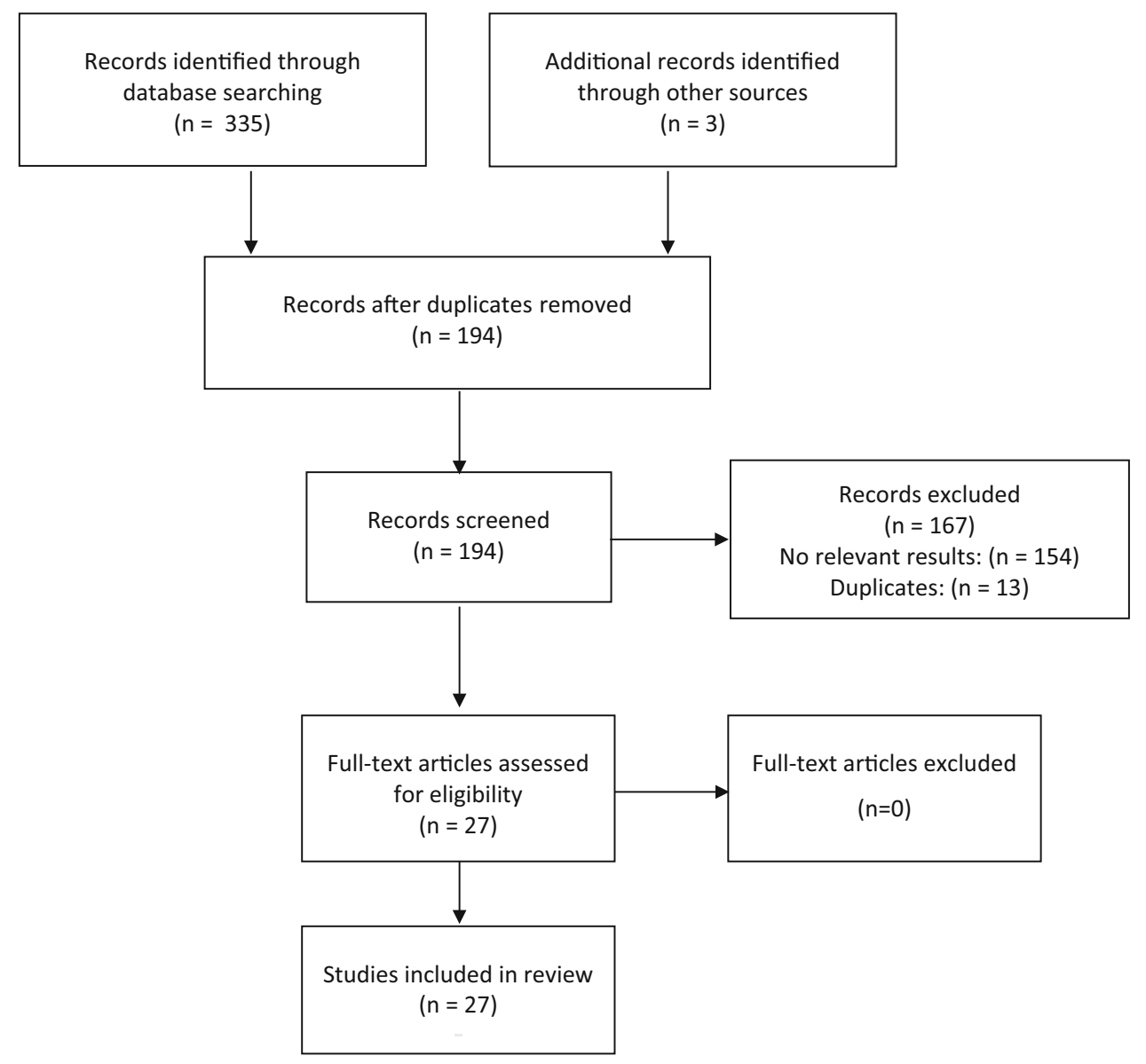

Fig. 1 PRISMA diagram of results of search strategy

Most studies, irrespective of whether database analyses [42-46, 48] or clinical studies $[13,34,36-39,41]$ reported fever in women who received pertussis vaccination during pregnancy; the incidence ranged from $0 \%$ to $5.1 \%$ in Tdap vaccinated pregnant women (Table 1). Kharbanda et al. [42] found that Tdapvaccinated pregnant women were 5.4-times more likely to experience medically attended (i.e. requiring a visit to a health care professional) fever in the 3 days post-vaccination than unvaccinated pregnant women in a large database analysis (adjusted incident rate ratio [AIRR] $=5.4$ [95\% CI 2.1-13.9]). In contrast, a small clinical study by Munoz et al. [13] showed that the frequency of fever in Tdap-vaccinated pregnant women was comparable with that following placebo, and to that among Tdapvaccinated non-pregnant women, but lower than that in women vaccinated postpartum. A factor potentially contributing to differences in the reported rate of fever was the timing of vaccination. The rate of fever appears lower in women vaccinated $\geq 27$ weeks' gestation than in those vaccinated earlier $(<27$ weeks' gestation) or within 7 days postpartum (3.80 per 10,000 pregnant women vs. 5.19 per 10,000 and 11.69 per 10,000 women, respectively) [43]. Database analyses and clinical studies that reported other systemic adverse events following Tdap vaccination during pregnancy, such as headache, nausea/vomiting, malaise, myalgia and fatigue, found that pregnancy did not increase the reported rate of these adverse events [13, 34, 36-38, 40, 42].

The reactogenicity events reported were predominantly mild and self-limiting. Two clinical studies reported that either no [41] or $<2 \%$ [34] of pregnant women experienced serious injection site reactions after Tdap 
and $<1 \%$ experienced serious systemic symptoms [34]. Across individual reactogenicity symptoms, $<20 \%$ of pregnant women experienced moderate-to-severe events, commonly injection site pain or tenderness [34, 41].

Concerns about the safety of repeat vaccination with Tdap (or in women who had previously received a tetanus-containing vaccine) at sometimes short intervals between successive pregnancies persist [49]. Regan et al. [39] reported a trend towards increased odds of several adverse event in women with a history of Tdap vaccination 3 years before the current Tdap vaccination during pregnancy (in 2011-2012 vs. current year 2015), but myalgia was the only specific event reported with significantly increased odds [odd ration; 4.92 (1.11-21.83)]. The frequency of systemic and local reactions, including fever, among Tdapvaccinated pregnant women who had received a dose of tetanus-containing vaccine within 1-5 years previously was similar to that in those who had no such vaccinations in two clinical studies [34, 41]. In addition, the rate of acute adverse events reported in a database analysis, among pregnant women who received a tetanus-containing vaccine $<2$ years and $2-5$ years was similar to that reported in those vaccinated $>5$ years previously [45].

The safety of concomitant maternal vaccination with Tdap and influenza vaccines during pregnancy was assessed in two studies [39, 45]. Regan et al. [39] found no evidence of enhanced safety risks with concomitant Tdap and trivalent influenza vaccination compared with Tdap vaccination, as reported by pregnant women using a variety of reactogenicity measures (Table 1). A large US database review found no differences in the frequency of medically attended fever or any acute reaction between pregnant women who received Tdap and influenza vaccines concomitantly compared with sequentially [45].

\section{Maternal Adverse Events and Adverse Fetal Outcomes Pre-delivery}

Twenty-one publications were identified that assessed maternal adverse events in women who received Tdap during pregnancy and pregnancy outcomes (Table 1). Of these, 15 were database analyses [42-48, 50-57]. Chorioamnionitis was identified as slightly increased in pregnant women who received Tdap (relative risk 1.19; 95\% confidence interval [CI] 1.13-1.26) in a 2010-2012 retrospective, observational, cohort study using data from two Vaccine Safety Datalink sites [54]. Chorioamnionitis increased by a marginally lesser extent in women vaccinated between 27 and 36 gestational weeks (1.11; 95\% CI 1.03-1.21). However, pre-term birth (a major chorioamnionitis sequela) was not increased. Subsequently, similar results were reported from database analyses by Layton et al. [43] and DeSilva et al. [52]; both analyses found small but significant increases in chorioamnionitis in women vaccinated with Tdap during pregnancy compared with unvaccinated pregnant women $[43,52]$. In contrast, other database analyses found no significant difference in chorioamnionitis between women who did and did not receive the Tdap vaccine during pregnancy $[48,50,55]$, including those who received $\geq 2$ Tdap doses in the past 5 years [55]. An analysis of 31 chorioamnionitis cases among 3389 pregnancy reports in the Vaccine Adverse Event Reporting System (VAERS) database (between 1990 and 2014) identified that $58 \%$ of cases were in women with pre-existing risk factors [51]. Tdap (26\%), H1N1 inactivated influenza vaccine (32\%) and the quadrivalent human papillomavirus vaccine (29\%) accounted for the majority of chorioamnionitis cases reported to the VAERS.

Other pregnancy outcomes, including preeclampsia/eclampsia, intrauterine growth retardation, premature labor/contraction, postpartum hemorrhage, placenta previa, elective and spontaneous abortions, oligohydramnios, proteinuria, venous thromboembolism, cardiac events, and gestational hypertensive disorder have also been evaluated. These adverse events were not significantly increased among Tdapvaccinated vs. non-vaccinated pregnant women across multiple database analyses $[42,43,47,48,50,53-56]$ and clinical studies $[13,41,58]$, even when consecutive doses of Tdap vaccine were repeated at intervals of $<5$ years [55]. Although Layton et al. [43] 
found a small increase in postpartum hemorrhage in Tdap vaccinated compared with unvaccinated pregnant women $(\mathrm{RR}=1.23 ; 95 \%$ CI 1.18-1.28) [43], the database analyses of Donegan et al. [53] and Griffin et al. [48] found no such increase with vaccination. "Serious" adverse pregnancy outcomes occurred at similar rates in Tdap- and Td-vaccinated pregnant women in a randomized prospective trial [35], and in Tdap plus influenza sequentially- or concomitantly-vaccinated pregnant women identified in an other database analysis [45].

There was no causal association between the Tdap vaccine and adverse outcomes reported in several clinical studies [13, 37, 38, 41].

\section{Adverse Birth and Neonatal Outcomes/ Complications}

Concerns regarding potential adverse birth and neonatal outcomes can influence decisions and recommendations about Tdap vaccination during pregnancy. Outcomes assessed included preterm birth, stillbirth, development-related parameters such as low/very low birth weight or small size for gestational age, neonatal death, birth defects (including atrioventricular septal defect, spina bifida, cleft palate, cleft lip and diaphragmatic hernia), major malformations, congenital anomalies, neonatal complications, respiratory disorders, APGAR score, and cord blood $\mathrm{pH}$ values (Table 1). No significant increase in any of these outcomes were identified among infants of Tdap-vaccinated compared to non-vaccinated pregnant women, or from expected norms, regardless of design (database analysis vs. clinical trial), population, or setting $[13,41,43,50,52-56,58]$. In addition, no differences in the rates of serious adverse outcomes were observed in infants of Tdap- and Td-vaccinated pregnant women, and none of the serious adverse outcomes reported were considered related to vaccination [35]. Hoang et al. [36] reported that infants of Tdapvaccinated women presented common symptoms of respiratory and gastrointestinal diseases, although no events were serious or considered related to vaccination. Furthermore, Tdap vaccination during pregnancy does not appear to be associated with an increase in the diagnosis of autism spectrum disorder [59].

There was no increase in acute adverse events or adverse birth outcomes in infants of women who received Tdap vaccination during pregnancy following previous vaccination with a tetanus-containing vaccine $<2$ years or $2-5$ years before compared with those whose mothers had been vaccinated $>5$ years [45]. Nor between infants of women who had received single vs. multiple $(<5$ years) doses of Tdap [55]. The timing of Tdap relative to influenza vaccine in had no influence on the frequency of preterm delivery, low birth weight and small for gestational age in infants of women who received sequential or concomitant immunization with these vaccines during pregnancy [46].

Infants of women who received Tdap during pregnancy did not need more or more intense healthcare support than those from women who did not receive the vaccine, with no increases recorded in the days of neonatal hospitalization or neonatal intensive care unit (NICU) admission $[50,55]$, or in the number of health encounters with complex chronic conditions by age 12 months [56]. In addition, there was no significant association between infant hospitalization or death in the first 6 months of life and receipt of maternal Tdap or Tdap plus influenza vaccine [60].

\section{DISCUSSION}

Concerns about maternal pertussis vaccination, at a time when the mother is cautious about their own health and that of their unborn child, may adversely influence acceptance and uptake of the vaccine. This review confirms the safety of maternal pertussis vaccination during pregnancy, and provides evidence to support decision-making and counseling on this intervention. Although local and systemic reactions were reported among vaccinated pregnant women in clinical studies and database analyses, these were usually mild and selflimiting and generally well tolerated. Such reactions were not influenced by repeat exposure to Tdap, vaccines containing tetanus 
toxoid or by concomitant vaccination with influenza vaccines (Table 1 ).

This review provides reassurance that vaccination against pertussis during pregnancy does not adversely affect maternal, fetal or neonatal health (Table 1). Although the studies reviewed used different methodologies and reporting measures, they were generally in agreement. However, in the future, it may be possible to better synthesize the available data if initiatives, such as that of the Global Alignment of Immunization Safety Assessment in Pregnancy (GAIA) project, coordinated by the Brighton Collaboration Foundation [61], are adopted. The GAIA project was initiated to provide guidance for harmonizing the data collected in case report forms used for safety monitoring, with the aim of improving data collection and understanding of vaccine-related outcomes in pregnancy.

The potential impact of Tdap vaccination during pregnancy on chorioamnionitis varies, with some studies suggesting an increased rate of the condition $[43,52,54]$ and others no such increase $[48,50,55]$. Nonetheless, chorioamnionitis is uncommonly reported ( $1 \%$ of pregnancy reports to VAERS databases), and usually observed in pregnant women with known risk factors for the condition [51].

There is currently no definitive reactogenicity data by trimester, or on pregnancy, birth and neonatal outcomes. In Ireland, the National Immunization Advisory Committee (NIAC) recommended (in 2016) that pregnant women should be given the vaccine as early as 16 weeks' gestational [62]. This advice was based on a study [63] which found that the immune response was robust when the vaccine was given early in second trimester. The extended eligibility criteria for the vaccine in the UK made the vaccine available to women beginning in the sixteenth week of pregnancy since April 2016 (previously available from 28 weeks), and may have contributed to the increase in the coverage during pregnancy [64]. Examination of the impact of the earlier vaccination during pregnancy will be informative.

In recent years, many countries including the UK, USA, Australia, New Zealand, and several others in Europe and Latin America have introduced pertussis vaccination during pregnancy. Of note, this review included data from most of these countries and regions. There were no apparent geographic differences in the safety profile of maternal pertussis immunization during pregnancy and on infant outcomes.

Our systematic review has a number of limitations that need to be considered. Despite a rigorous systematic search to identify all available articles published between 1995 and 2018, we cannot exclude the possibility of missing some studies and nor did we assess publication bias. Our broad inclusion criteria resulted in a diverse range of included studies which complicates the overall interpretation of results as there was no standardized reporting of adverse events/complications. In addition, a large proportion of the articles reviewed were from the USA. The strength of our review lies in our general adherence to established methods for conducting systematic reviews, including an extensive search across multiple databases, and a wide inclusive publication date range.

\section{CONCLUSION}

In conclusion, our review consolidates knowledge on the safety profile of maternal pertussis immunization during pregnancy and on the infant. Maternal immunization does not adversely affect pregnancy, birth or neonatal outcomes. Evidence presented confirms the safety of maternal pertussis immunization, and supports recommendations for pertussis vaccination during pregnancy.

\section{ACKNOWLEDGEMENTS}

Funding. This review and the journal's rapid service fee were funded by Sanofi Pasteur,

Medical Writing and Editorial Assistance. Editorial assistance with the preparation of the manuscript was provided by Caroline Spencer on behalf of inScience Communications, Springer Healthcare, UK, and was funded by Sanofi Pasteur. The authors also thank 
Burnedette Rose Hill for editorial assistance and manuscript coordination on behalf of SanofiPasteur.

Authorship. All named authors meet the International Committee of Medical Journal Editors (ICMJE) criteria for authorship for this article, take responsibility for the integrity of the work as a whole, and have given their approval for this version to be published.

Disclosures. Charlotte Switzer is an employee of Sanofi Pasteur. Caroline D'Heilly is an employee of Sanofi Pasteur. Denis Macina is an employee of Sanofi Pasteur.

Compliance with Ethics Guidelines. This article is based on previously conducted studies and does not contain any studies with human participants or animals performed by any of the authors.

Open Access. This article is distributed under the terms of the Creative Commons Attribution-NonCommercial 4.0 International License (http://creativecommons.org/licenses/ by-nc/4.0/), which permits any noncommercial use, distribution, and reproduction in any medium, provided you give appropriate credit to the original author(s) and the source, provide a link to the Creative Commons license, and indicate if changes were made.

\section{REFERENCES}

1. Edwards KM, Decker MD. Pertussis vaccines. In: Plotkin SA, Orenstein WA, Offit PA, Edwards KM, editors. Plotkin's vaccines. 7th ed. Philadelphia: Elsevier; 2018. p. 711-61.

2. Yeung KHT, Duclos P, Nelson EAS, Hutubessy RCW. An update of the global burden of pertussis in children younger than 5 years: a modelling study. Lancet Infect Dis. 2017;17(9):974-80.

3. WHO. Pertussis vaccines: WHO position paperSeptember 2015. Wkly Epidemiol Rec. 2015;90(35):433-58.

4. Bento AI, King AA, Rohani P. Maternal pertussis immunisation: clinical gains and epidemiological legacy. Euro Surveill. 2017;22:15.
5. Poolman JT. Shortcomings of pertussis vaccines: why we need a third generation vaccine. Expert Rev Vaccines. 2014;13(10):1159-62.

6. Skoff TH, Kenyon C, Cocoros N, Liko J, Miller L, Kudish $\mathrm{K}$, et al. Sources of infant pertussis infection in the United States. Pediatrics. 2015;136(4):635-41.

7. Wiley KE, Zuo Y, Macartney KK, McIntyre PB. Sources of pertussis infection in young infants: a review of key evidence informing targeting of the cocoon strategy. Vaccine. 2013;31(4):618-25.

8. Masseria C, Martin CK, Krishnarajah G, Becker LK, Buikema A, Tan TQ. Incidence and burden of pertussis among infants less than 1 year of age. Pediatr Infect Dis J. 2017;36(3):e54-61.

9. Romanin V, Agustinho V, Califano G, Sagradini S, Aquino A, Juarez Mdel V, et al. Epidemiological situation of pertussis and strategies to control it: Argentina, 2002-2011. Arch Argent Pediatr. 2014;112(5):413-20.

10. Torres RS, Santos TZ, Torres RA, Pereira VV, Favero LA, Otavio Filho RM, et al. Resurgence of pertussis at the age of vaccination: clinical, epidemiological, and molecular aspects. J Pediatr (Rio J). 2015;91(4):333-8.

11. van Hoek AJ, Campbell $H$, Amirthalingam G, Andrews N, Miller E. The number of deaths among infants under one year of age in England with pertussis: results of a capture/recapture analysis for the period 2001 to 2011. Euro Surveill. 2013;18:9.

12. Esposito S, Principi N. Immunization against pertussis in adolescents and adults. Clin Microbiol Infect. 2016;22(Suppl 5):S89-95.

13. Munoz FM, Bond NH, Maccato M, Pinell P, Hammill HA, Swamy GK, et al. Safety and immunogenicity of tetanus diphtheria and acellular pertussis (Tdap) immunization during pregnancy in mothers and infants: a randomized clinical trial. JAMA. 2014;311(17):1760-9.

14. Amirthalingam G, Andrews N, Campbell H, Ribeiro S, Kara E, Donegan K, et al. Effectiveness of maternal pertussis vaccination in England: an observational study. Lancet. 2014;384(9953):1521-8.

15. Amirthalingam G, Campbell H, Ribeiro S, Fry NK, Ramsay M, Miller E, et al. sustained effectiveness of the maternal pertussis immunization program in England 3 years following introduction. Clin Infect Dis. 2016;63(suppl 4):S236-43.

16. Baxter R, Bartlett J, Fireman B, Lewis E, Klein NP. Effectiveness of vaccination during pregnancy to prevent infant pertussis. Pediatrics. 2017;139:5. 
17. Dabrera G, Amirthalingam G, Andrews N, Campbell H, Ribeiro S, Kara E, et al. A case-control study to estimate the effectiveness of maternal pertussis vaccination in protecting newborn infants in England and Wales, 2012-2013. Clin Infect Dis. 2015;60(3):333-7.

18. Romanin V, Acosta AM, Sagradini S, Briere E, Sanchez SM, Sevilla ME, et al. Effectiveness of Tdap vaccination during pregnancy in preventing infant pertussis in a country with whole-cell pertussis vaccines during childhood: preliminary results of a case-control study in Argentina. Open Forum Infect Dis. 2015;2(Suppl 1):S447.

19. Saul N, Wang K, Bag S, Baldwin H, Alexander K, Chandra $\mathrm{M}$, et al. Effectiveness of maternal pertussis vaccination in preventing infection and disease in infants: the NSW Public Health Network case-control study. Vaccine. 2018;36(14):1887-92.

20. Skoff TH, Blain AE, Watt J, Scherzinger K, McMahon M, Zansky SM, et al. Impact of the US maternal tetanus, diphtheria, and acellular pertussis vaccination program on preventing pertussis in Infants $<2$ months of age: a case-control evaluation. Clin Infect Dis. 2017;65(12):1977-83.

21. Winter K, Nickell S, Powell M, Harriman K. Effectiveness of prenatal versus postpartum tetanus, diphtheria, and acellular pertussis vaccination in preventing infant pertussis. Clin Infect Dis. 2017;64(1):3-8.

22. Winter K, Cherry JD, Harriman K. Effectiveness of prenatal tetanus, diphtheria, and acellular pertussis vaccination on pertussis severity in infants. Clin Infect Dis. 2017;64(1):9-14.

23. Bonville CA, Cibula DA, Domachowske JB, Suryadevara M. Vaccine attitudes and practices among obstetric providers in New York State following the recommendation for pertussis vaccination during pregnancy. Hum Vaccin Immunother. 2015;11(3):713-8.

24. Chamberlain AT, Seib K, Ault KA, Orenstein WA, Frew PM, Malik F, et al. Factors associated with intention to receive influenza and tetanus, diphtheria, and acellular pertussis (Tdap) vaccines during pregnancy: a focus on vaccine hesitancy and perceptions of disease severity and vaccine safety. PLoS Curr. 2015;2015:7.

25. Wilson RJ, Paterson P, Jarrett C, Larson HJ. Understanding factors influencing vaccination acceptance during pregnancy globally: a literature review. Vaccine. 2015;33(47):6420-9.

26. Jones KM, Carroll S, Hawks D, McElwain CA, Schulkin J. Efforts to improve immunization coverage during pregnancy among Ob-Gyns. Infect Dis Obstet Gynecol. 2016;2016:6120701.

27. Vishram B, Letley L, Jan Van Hoek A, Silverton L, Donovan H, Adams C, et al. Vaccination in pregnancy: attitudes of nurses, midwives and health visitors in England. Hum Vaccin Immunother. 2018;14(1):179-88.

28. Wilcox CR, Bottrell K, Paterson P, Schulz WS, Vandrevala $\mathrm{T}$, Larson $\mathrm{HJ}$, et al. Influenza and pertussis vaccination in pregnancy: portrayal in online media articles and perceptions of pregnant women and healthcare professionals. Vaccine. 2018;36(50):7625-31.

29. Maisa A, Milligan S, Quinn A, Boulter D, Johnston J, Treanor $\mathrm{C}$, et al. Vaccination against pertussis and influenza in pregnancy: a qualitative study of barriers and facilitators. Public Health. 2018;162:111-7.

30. Collins J, Alona I, Tooher R, Marshall H. Increased awareness and health care provider endorsement is required to encourage pregnant women to be vaccinated. Hum Vaccin Immunother. 2014;10(10):2922-9.

31. Mehrotra A, Fisher AK, Mullen J, Rodriguez L, Jiles AJ, Albert AP, et al. Provider insight on surmounting specialty practice challenges to improve Tdap immunization rates among pregnant women. Heliyon. 2018;4(5):e00636.

32. Switzer C, Macina D. Safety and effectiveness of pertussis immunization during pregnancy 2016 Contract No: CRD42016038317.

33. O'Connor D, Green S, Higgins JPT. Chapter 5: Defining the review question and developing criteria for including studies. Cochrane Handbook for Systematic Reviews of Interventions. Version 5.0.0 ed2008 ed2008.

34. Fortner KB, Swamy GK, Broder KR, Jimenez-Truque $\mathrm{N}$, Zhu Y, Moro PL, et al. Reactogenicity and immunogenicity of tetanus toxoid, reduced diphtheria toxoid, and acellular pertussis vaccine (Tdap) in pregnant and nonpregnant women. Vaccine. 2018;36(42):6354-60.

35. Halperin SA, Langley JM, Ye L, MackinnonCameron D, Elsherif $M$, Allen VM, et al. A randomized controlled trial of the safety and immunogenicity of tetanus, diphtheria, and acellular pertussis vaccine immunization during pregnancy and subsequent infant immune response. Clin Infect Dis. 2018;67(7):1063-71.

36. Hoang HT, Leuridan E, Maertens K, Nguyen TD, Hens N, Vu NH, et al. Pertussis vaccination during pregnancy in Vietnam: results of a randomized 
controlled trial Pertussis vaccination during pregnancy. Vaccine. 2016;34(1):151-9.

37. Maertens K, Cabore RN, Huygen K, Hens N, Van Damme P, Leuridan E. Pertussis vaccination during pregnancy in Belgium: results of a prospective controlled cohort study. Vaccine. 2016;34(1):142-50.

38. Petousis-Harris H, Walls T, Watson D, Paynter J, Graham P, Turner N. Safety of Tdap vaccine in pregnant women: an observational study. BMJ Open. 2016;6(4):e010911.

39. Regan AK, Tracey LE, Blyth CC, Richmond PC, Effler PV. A prospective cohort study assessing the reactogenicity of pertussis and influenza vaccines administered during pregnancy. Vaccine. 2016;34(20):2299-304.

40. Villarreal Perez JZ, Ramirez Aranda JM, de la Manuel OC, Zamudio Osuna MJ, Perales Davila J, Ballesteros Elizondo MR, et al. Randomized clinical trial of the safety and immunogenicity of the Tdap vaccine in pregnant Mexican women. Hum Vaccin Immunother. 2017;13(1):128-35.

41. Wanlapakorn N, Maertens K, Chaithongwongwatthana S, Srimuan D, Suratannon N, Vongpunsawad S, et al. Assessing the reactogenicity of Tdap vaccine administered during pregnancy and antibodies to Bordetella pertussis antigens in maternal and cord sera of Thai women. Vaccine. 2018;36(11):1453-9.

42. Kharbanda EO, Vazquez-Benitez G, Lipkind HS, Klein NP, Cheetham TC, Naleway AL, et al. Maternal Tdap vaccination: coverage and acute safety outcomes in the vaccine safety datalink, 2007-2013. Vaccine. 2016;34(7):968-73.

43. Layton JB, Butler AM, Li D, Boggess KA, Weber DJ, McGrath LJ, et al. Prenatal Tdap immunization and risk of maternal and newborn adverse events. Vaccine. 2017;35(33):4072-8.

44. Moro PL, Cragan J, Tepper N, Zheteyeva Y, Museru $\mathrm{O}$, Lewis $\mathrm{P}$, et al. Enhanced surveillance of tetanus toxoid, reduced diphtheria toxoid, and acellular pertussis (Tdap) vaccines in pregnancy in the Vaccine Adverse Event Reporting System (VAERS), 2011-2015. Vaccine. 2016;34(20):2349-53.

45. Sukumaran L, McCarthy NL, Kharbanda EO, McNeil MM, Naleway AL, Klein NP, et al. Association of Tdap vaccination with acute events and adverse birth outcomes among pregnant women with prior tetanus-containing immunizations. JAMA. 2015;314(15):1581-7.

46. Sukumaran L, McCarthy NL, Kharbanda EO, Weintraub ES, Vazquez-Benitez G, McNeil MM, et al. Safety of tetanus toxoid, reduced diphtheria toxoid, and acellular pertussis and influenza vaccinations in pregnancy. Obstet Gynecol. 2015;126(5):1069-74.

47. Zheteyeva YA, Moro PL, Tepper NK, Rasmussen SA, Barash FE, Revzina NV, et al. Adverse event reports after tetanus toxoid, reduced diphtheria toxoid, and acellular pertussis vaccines in pregnant women. Am J Obstet Gynecol. 2012;207(1):59 e1-7.

48. Griffin JB, Yu L, Watson D, Turner N, Walls T, Howe AS, et al. Pertussis Immunisation in Pregnancy Safety (PIPS) Study: a retrospective cohort study of safety outcomes in pregnant women vaccinated with Tdap vaccine. Vaccine. 2018;36(34):5173-9.

49. Advisory Committee on Immunization Practices (ACIP), Updated recommendations for use of tetanus toxoid, reduced diphtheria toxoid, and acellular pertussis vaccine (Tdap) in pregnant women-Advisory Committee on Immunization Practices (ACIP). MMWR Morb Mortal Wkly Rep. 2013;62(7):131-5.

50. Berenson $\mathrm{AB}$, Hirth JM, Rahman M, Laz TH, Rupp RE, Sarpong KO. Maternal and infant outcomes among women vaccinated against pertussis during pregnancy. Hum Vaccin Immunother. 2016;12(8):1965-71.

51. Datwani H, Moro PL, Harrington T, Broder KR. Chorioamnionitis following vaccination in the vaccine adverse event reporting system. Vaccine. 2015;33(27):3110-3.

52. DeSilva M, Vazquez-Benitez G, Nordin JD, Lipkind HS, Klein NP, Cheetham TC, et al. Maternal Tdap vaccination and risk of infant morbidity. Vaccine. 2017;35(29):3655-60.

53. Donegan K, King B, Bryan P. Safety of pertussis vaccination in pregnant women in UK: observational study. BMJ. 2014;349:g4219.

54. Kharbanda EO, Vazquez-Benitez G, Lipkind HS, Klein NP, Cheetham TC, Naleway A, et al. Evaluation of the association of maternal pertussis vaccination with obstetric events and birth outcomes. JAMA. 2014;312(18):1897-904.

55. Morgan JL, Baggari SR, McIntire DD, Sheffield JS. Pregnancy outcomes after antepartum tetanus, diphtheria, and acellular pertussis vaccination. Obstet Gynecol. 2015;125(6):1433-8.

56. Shakib JH, Korgenski K, Sheng X, Varner MW, Pavia AT, Byington CL. Tetanus, diphtheria, acellular pertussis vaccine during pregnancy: pregnancy and infant health outcomes. $\mathrm{J}$ Pediatr. 2013;163(5):1422-1426 e1-4. 
57. Wang M, Khromava A, Mahmood A, Dickson N. Pregnant women receiving tetanus-diphtheriaacellular pertussis (Tdap) vaccine: 6 years of Adacel vaccine pregnancy registry data. Pharmacoepidemiol Drug Saf. 2011;20:S60.

58. Walls T, Graham P, Petousis-Harris H, Hill L, Austin $\mathrm{N}$. Infant outcomes after exposure to Tdap vaccine in pregnancy: an observational study. BMJ Open. 2016;6(1):e009536.

59. Becerra-Culqui TA, Getahun D, Chiu V, Sy LS, Tseng HF. Prenatal tetanus, diphtheria, acellular pertussis vaccination and autism spectrum disorder. Pediatrics. 2018;142:3.

60. Sukumaran L, McCarthy NL, Kharbanda EO, Vazquez-Benitez G, Lipkind HS, Jackson L, et al. Infant hospitalizations and mortality after maternal vaccination. Pediatrics. 2018;141:3.
61. Jones CE, Munoz FM, Kochhar S, Vergnano S, Cutland CL, Steinhoff M, et al. Guidance for the collection of case report form variables to assess safety in clinical trials of vaccines in pregnancy. Vaccine. 2016;34(49):6007-14.

62. National Immunisation Office. Whooping cough (Pertussis). Dublin: HSE National Immunisation Office; 2017.

63. Eberhardt CS, Blanchard-Rohner G, Lemaitre B, Boukrid M, Combescure C, Othenin-Girard V, et al. Maternal immunization earlier in pregnancy maximizes antibody transfer and expected infant seropositivity against pertussis. Clin Infect Dis. 2016;62(7):829-36.

64. Public Health England. Pertussis vaccination programme for pregnant women update: vaccine coverage in England, October to December 20162017. 